\title{
Improving menstrual hygiene management in emergency contexts: literature review of current perspectives
}

This article was published in the following Dove Press journal: International Journal of Women's Health

\author{
Crystal VanLeeuwen \\ Belen Torondel \\ Department of Disease Control, \\ London School of Hygiene and \\ Tropical Medicine, London, UK
}

Correspondence: Belen Torondel London School of Hygiene and Tropical Medicine, Kepple Street, London WCIE7HT, UK

Tel +442076362934

Fax +44 2079272739

Email belen.torondel@Ishtm.ac.uk

\begin{abstract}
Management of menstruation in contexts of humanitarian emergencies can be challenging. A lack of empirical research about effective interventions which improve menstrual hygiene management (MHM) among female populations in humanitarian emergencies and a lack of clarity about which sectors within a humanitarian response should deliver MHM interventions can both be attributable to the lack of clear guidance on design and delivery of culturally appropriate MHM intervention in settings of humanitarian emergencies. The objective of this review was to collate, summarize, and appraise existing peer-reviewed and gray literature that describes the current scenario of MHM in emergency contexts in order to describe the breadth and depth of current policies, guidelines, empirical research, and humanitarian aid activities addressing populations' menstrual needs. A structured-search strategy was conducted for peer-reviewed and gray literature to identify studies, published reports, guidelines, and policy papers related to menstrual response in emergency humanitarian contexts. Of the 51 articles included in the review, 16 were peer-reviewed papers and 35 were gray literature. Most of the literature agreed that hardware interventions should focus on the supply of adequate material (not only absorbent material but also other supportive material) and adequate sanitation facilities, with access to water and private space for washing, changing, drying, and disposing menstrual materials. Software interventions should focus on education in the usage of materials to manage menstruation hygienically and education about the female body's biological processes. There was clear agreement that the needs of the target population should be assessed before designing any intervention. Although there is insight about which factors should be included in an effective menstrual hygiene intervention, there is insufficient empirical evidence to establish which interventions are most effective in humanitarian emergencies and which sectors should be responsible for the coordination and implementation of such. Increased monitoring and evaluation studies of interventions should be completed and publicly shared, in order to feed evidence-based guidelines in the humanitarian sector.
\end{abstract} Keywords: menstrual hygiene, emergency, guidelines, evidence, public health

\section{Background}

\section{Menstrual hygiene as a public health issue}

Menstruation is a biological process that approximately half the world's population experiences for a significant period of their lifetime. Adequate menstrual hygiene management (MHM) has been defined as women and adolescent girls using clean menstrual material to absorb or collect menstrual blood. The girls must be able to change the absorbent in privacy as often as necessary for the duration of the menstrual cycle, using soap and water for washing the body as required, and they should have access to facilities to dispose of used menstrual management materials. ${ }^{1}$ 
In 2016, Sommer et $\mathrm{al}^{2}$ added aspects of education and dignity to this definition, expressing importance that females "understand the basic facts linked to the menstrual cycle and how to manage it with dignity and without discomfort or fear." Developments leading to the acceptance of MHM as a public health issue have been well lineated, ${ }^{3,4}$ with roots embedded in the educational impact on girls in low- and middle-income countries. Beyond the educational impact of MHM lay issues of gender-based violence, health outcomes, and human rights among vulnerable populations. With an increase in conflictrelated displacement in recent years, the consequences of inadequate access to menstrual hygiene $(\mathrm{MH})$ requirements should continue to be considered in humanitarian responses aimed at assisting these vulnerable populations.

\section{$\mathrm{MH}$ in emergency contexts}

In 2016, 10.3 million people were newly displaced, increasing the global total to 65.6 million individuals who had been forced from their homes. ${ }^{5}$ This number continues to grow significantly in 2017, as ongoing insecurities in Africa and the Middle East continue and new, massive displacement erupts in Southeast Asia. As populations flee insecurity, they are often forced to leave with few possessions, travel difficult routes, and live in precarious situations for significant periods of time. Biological processes do not generally cease during these times, and managing menstruation can be an additional challenge for displaced females. A loss of normal coping strategies, changes in socioeconomic status, being faced with new prioritization dilemmas, changes in the physical and social environment, and a lack of access to structural and material sanitation needs can compound MHM issues among displaced populations. It has been argued ${ }^{6}$ that MHM goes beyond water, sanitation, and hygiene (WASH) responses, and that a cross-sector approach must be taken to ensure that humanitarian actions meet women's MH needs in emergency contexts.

\section{Humanitarian response for improved $\mathrm{MH}$}

Menstrual needs have appeared in humanitarian aid discussions since 1994, and have become a more widely discussed topic in recent years, ${ }^{3,4,6}$ which has led to definition of the term "menstrual hygiene management" and identification of components of an adequate MHM response. ${ }^{1}$ The overarching goal of MHM interventions is to ensure that menstruating females are able to manage this healthy biological process in a sustainable, hygienic, and dignified manner. ${ }^{1,7}$ Sommer et $\mathrm{al}^{6}$ outlined the components of the holistic response required to improve MHM among women and girls in emergency settings, which included three essential components: access to appropriate menstrual material and additional supportive material for storage, washing, and drying; adequate infrastructure for water, sanitation, changing areas, and wastedisposal mechanisms; and menstrual health education and promotion. Continuous consultation with female beneficiaries during the intervention design and implementation phases overarch this holistic approach. ${ }^{8}$

Although interest and action toward providing appropriate means for MHM in humanitarian crises are gaining traction in the aid sector, several recent reviews of both academic and gray literature have highlighted significant gaps in examining $\mathrm{MH}$ in vulnerable populations. ${ }^{6,9-12}$ The most recent literature review by Sommer et $\mathrm{al}^{6}$ identified a lack of existing peer-reviewed evidence examining effective MHM approaches in emergencies. Despite improvements, a lack of clarity on the required key components of a complete MHM response and the most effective interventions to adapt in emergency contexts continued to exist ${ }^{6}$ until late 2017 with the release of a comprehensive MHM toolkit. ${ }^{8}$ Understanding the cultural beliefs and practices of populations, examining menstrual product appropriateness in specific contexts, adapting interventions adequately to the physical environment, proper coordination of actors, and clear guidance for monitoring and evaluating programs are among the gaps identified. In order to deliver efficient and effective interventions, there is a need for clear guidelines that capture beneficiaries' needs in differing contexts, with consideration of cultural practices, requirements for designing appropriate interventions, delivery systems, and monitoring and evaluation (M\&E) tools.

\section{Aim and objective}

This literature review aims to identify peer-reviewed and gray literature discussing MHM in emergency contexts with an objective of describing the breadth and depth of current policies, guidelines, empirical research, and humanitarian aid activities addressing populations' menstrual needs. The aim of the paper is not to provide a critical appraisal of the included studies, but a summary of issues for consideration, and consensus in approaches to addressing MHM in emergency contexts.

\section{Materials and methods}

A literature search was conducted between June 23 and October 23, 2017. Search terms were adapted according to the database, and included the terms "menstrual hygiene," "menstrua*," "refugee," "IDP," "displace*," "emergenc*," and "crisis." Four databases were searched: London School 
of Hygiene and Tropical Medicine Discover, ReliefWeb, Sustainable Sanitation Alliance, and Google Scholar. The London School of Hygiene and Tropical Medicine Discover database includes searches of the databases PubMed, CAB Abstracts, Embase, and Global Health via Ovid SP. References and authors of key documents were consulted for additional resources. See Table 1 for details of search terms by database.

Inclusion criteria and restrictions set for this search were as follows:

- restricted to English language

- restricted to emergency contexts and/or broad, crosscontext documents

- no date restriction

- no study-participant age restriction

- no restriction on study or publication type.

\section{Results}

The literature search did not include a date limit to ensure as wide a range of articles were identified as possible, but it included all articles found up until October 23, 2017. In total, 1,202 documents were returned in an initial search and hand searching providing an additional nine entries. Exclusion of 1,055 documents which did not show any relevance to the research topic occurred after reviewing titles and abstract. The remaining 147 documents were more closely examined, and 75 were rejected for a focus on subjects unrelated to MHM in emergency humanitarian contexts. Articles containing only minor points quoted from primary sources already returned in the search were also rejected. In total, 51 documents were included in this review. See Figure 1 for a PRISMA (Preferred Reporting Items for Systematic Reviews and Meta-analyses) flowchart of search results.

\section{Description of articles included in the review}

Of the 51 articles included in the review, 16 were peer-reviewed papers, 35 were gray literature. Nine of the peer-reviewed

Table I Literature-search terms by database

LSHTM Discover

- (Menstrual hygiene or MHM) AND (refugee or displace* or emergenc* or crisis)

ReliefWeb

- Menstrual hygiene

Sustainable Sanitation Alliance Library

- Menstrual hygiene

Google Scholar

- "Menstrual hygiene" AND "emergency"

Abbreviations: LSHTM, London School of Hygiene and Tropical Medicine; MHM, menstrual hygiene management.

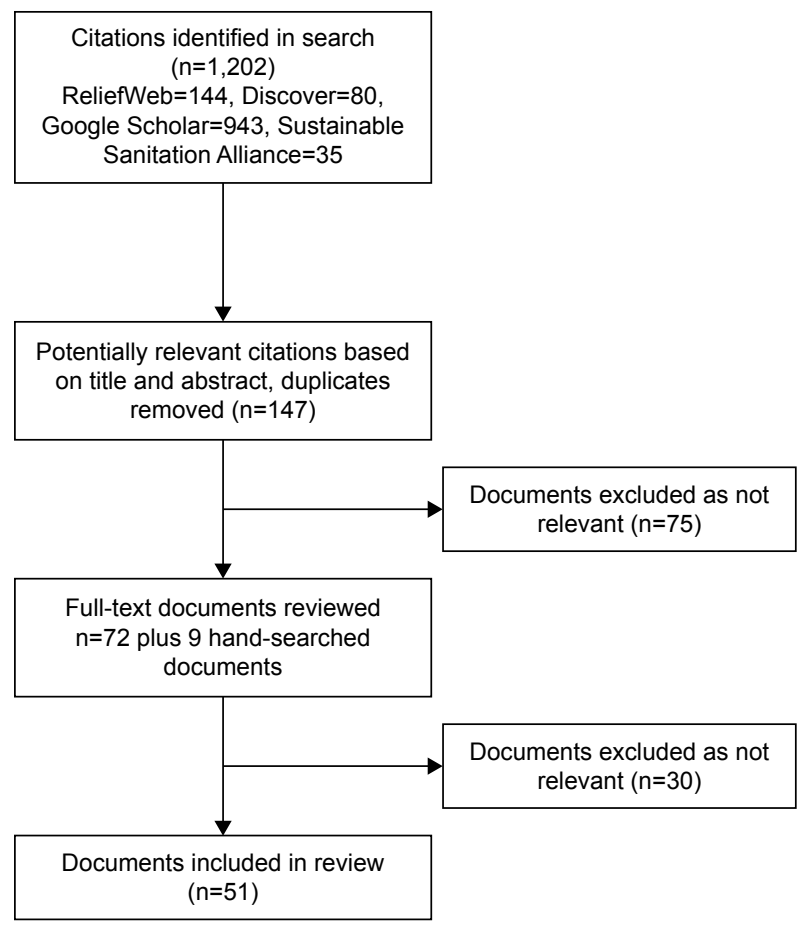

Figure I PRISMA flowchart of search. Abbreviation: PRISMA, Preferred Reporting Items for Systematic Reviews and Meta-analyses.

papers (see Table 2) were original research studies and seven were literature reviews (not shown in tables). From the gray literature, 4 were master's theses, 11 guidelines (see Table 3), and 20 nongovernmental organization (NGO) reports (communications, policy briefs, evaluations, and case studies).

From the seven reviews found in the search, only two focused on MHM in emergency contexts, ${ }^{4,6}$ four included some component of MHM, mainly within water, hygiene and sanitation (WASH) in emergency contexts, ${ }^{10,13-15}$ and one include MHM within the reproductive health and protection sectors in a humanitarian context. ${ }^{16}$ Literature found in this review has been grouped into six broad categories: supportive menstrual materials, sanitation infrastructure, knowledge and education, health, social context, gaps between policy and practice, and current guidelines.

\section{Supportive menstrual materials}

What is likely the most obvious and thus the most widely articulated requirement for $\mathrm{MHM}$ is ensuring access to appropriate absorbent and supportive menstrual materials. Absorbent materials, underwear, soap, and culturally appropriate storage spaces were mentioned in different papers as required MHM materials. ${ }^{17,20-22,24,26}$ Government subsidization and tax reduction/removal on menstrual products were 


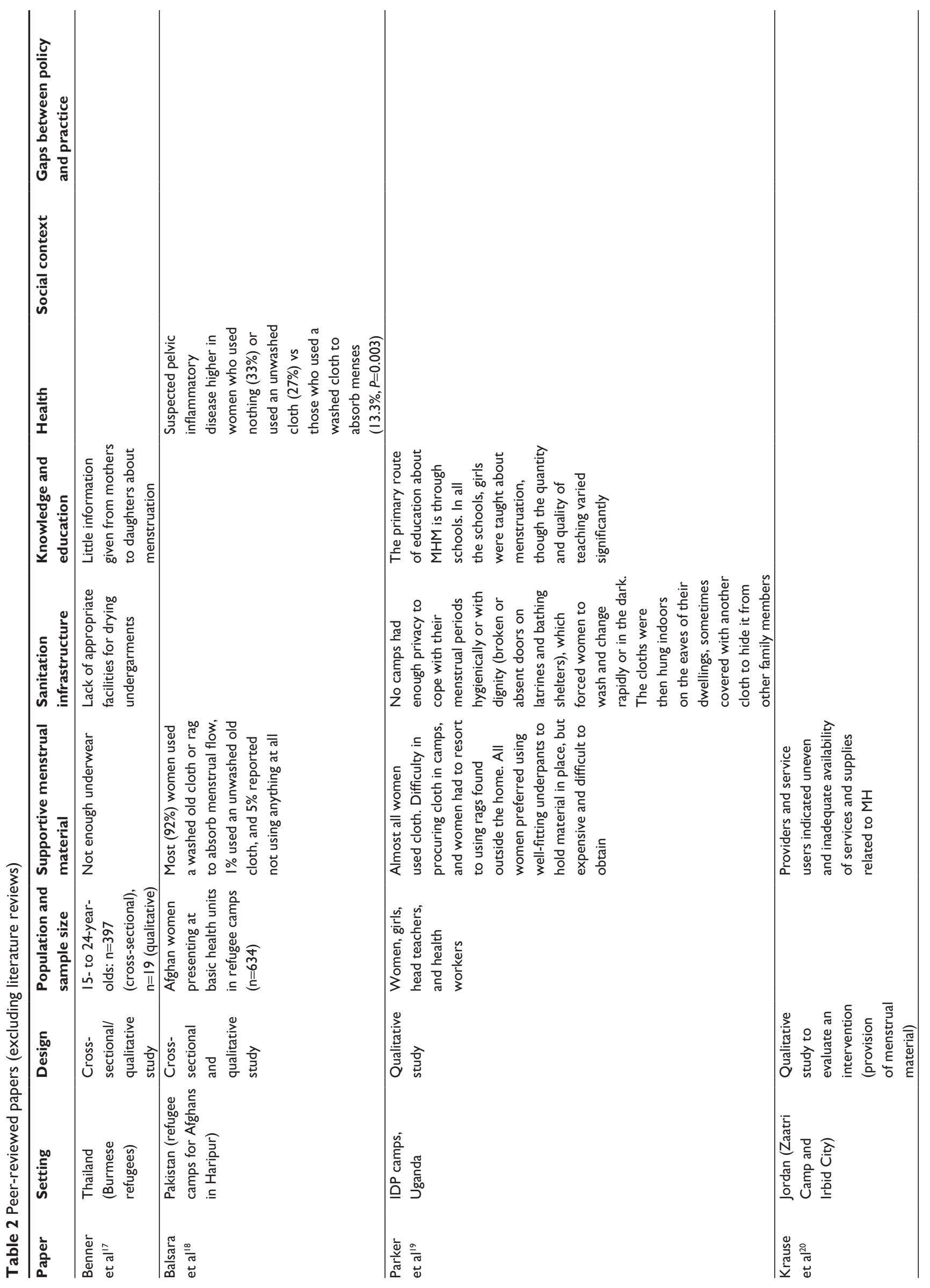




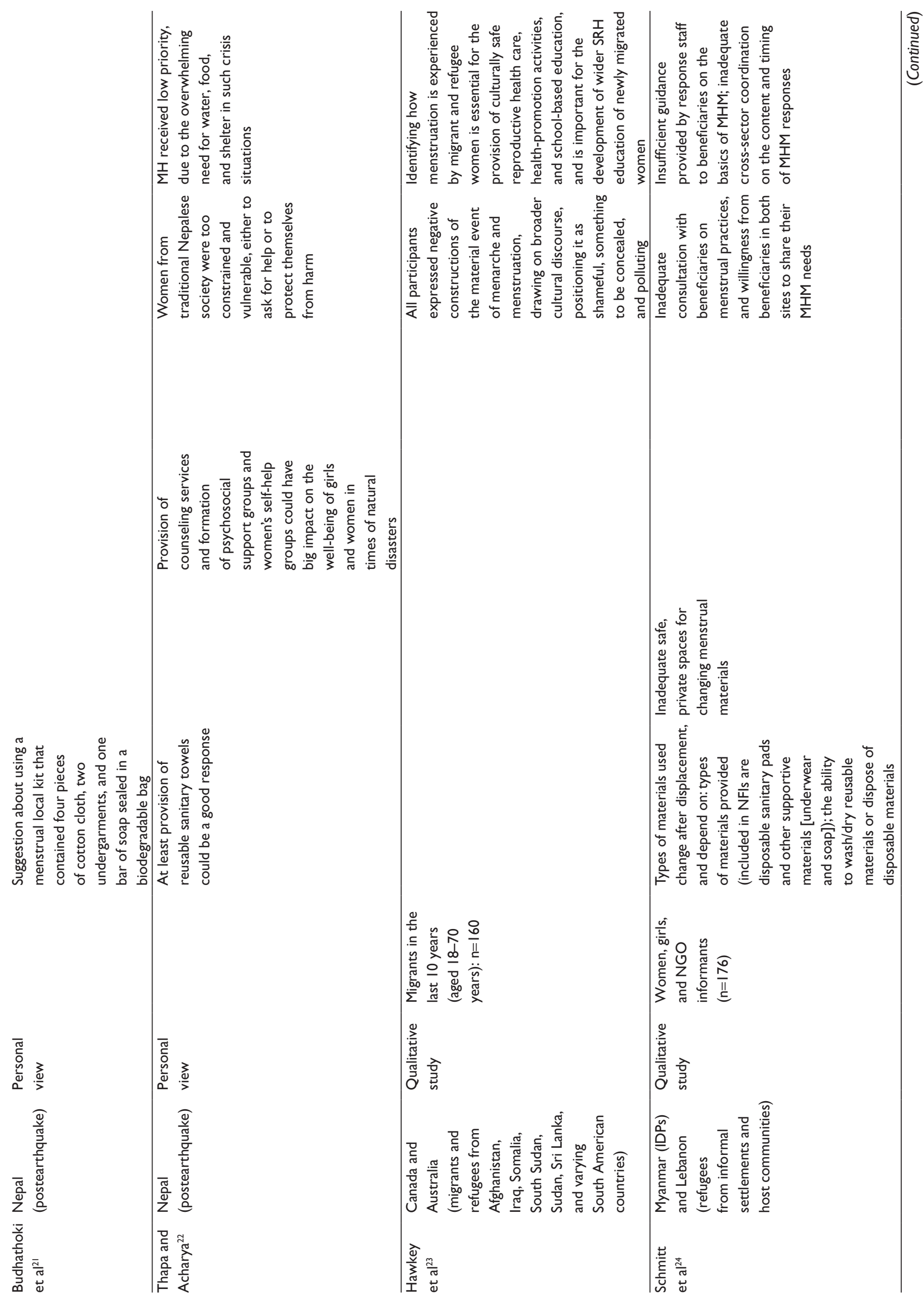




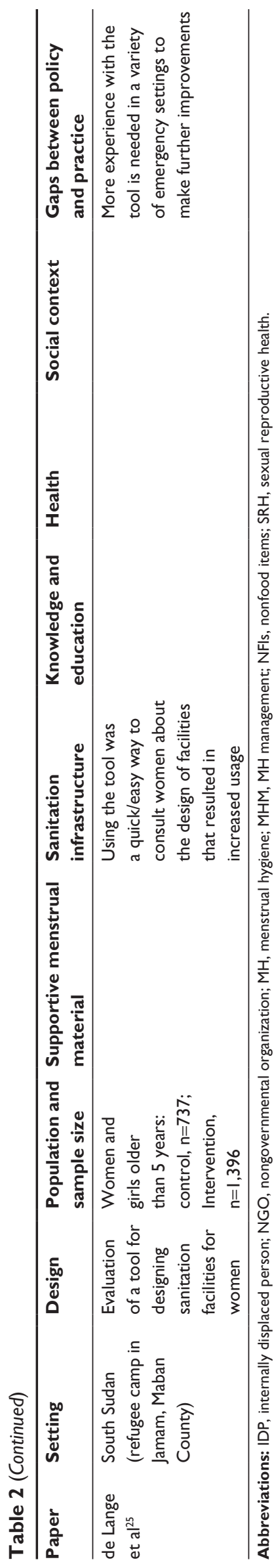

also mentioned strategies for improving access to required absorbents. ${ }^{12,27}$ Humanitarian interventions include provision of MH materials through distribution of individual items or in the form of "dignity kits," ${ }^{20-22,24}$ or the provision of cash or vouchers for individual purchasing.

Dignity kits, which were piloted in 2012, are basic hygiene items packaged for distribution, and are reported to alleviate difficult purchasing decisions associated with low resources and competing priorities that populations often face in emergency contexts. ${ }^{28}$ The need to evaluate the appropriateness of dignity-kit contents in differing contexts was expressed, ${ }^{4}$ and should also consider the available sanitation infrastructure. A document provided by the United Nations Population Fund ${ }^{29}$ was found succinctly to outline culturally appropriate $\mathrm{MH}$ items for distribution in the Myanmar context, as well as considerations for method of distribution and hygiene-promotion activities. Budhathoki et $\mathrm{al}^{21}$ suggested using a local menstrual kit in Nepal that contained four pieces of cotton cloth, two undergarments, and one bar of soap sealed in a biodegradable bag.

A recent review of Oxfam programs ${ }^{30}$ reported that cash programs were found to be more likely to contribute to restoring dignity in displaced populations and that there was little evidence of neglected hygiene-related purchasing through the use of cash models. The author went on to reiterate that impact must be monitored to ensure that women were able to manage menstruation in an adequate manner.

The choice of menstrual product is an individual decision that may be influenced by culture, religion, taboos, convenience, access, knowledge, socioeconomic status, and the availability of WASH materials and infrastructure. ${ }^{9,12,15,31}$ Schmitt et $\mathrm{al}^{24}$ mentioned that the types of menstrual absorbents used by displaced girls and women tended to change after displacement due to the aforementioned factors, and suggested that more work be done on assessing the needs and context of each setting before designing an MHM intervention. In this specific example, NGOs were distributing disposable menstrual materials to women coming from rural areas from Myanmar who normally used reusable material.

Product choice comes with economic and environmental impact at both the individual and global levels. ${ }^{7}$ Strategies aimed at making menstrual materials more affordable, accessible, and less damaging to the environment have emerged in recent years, ,7,27,32-34 although gaps in research examining the development of innovative, sustainable, and affordable products continue to exist. ${ }^{27}$ Reusable menstrual pads, menstrual underwear, and menstrual cups are viewed as sustainable and ecologically friendly options, although not often available 


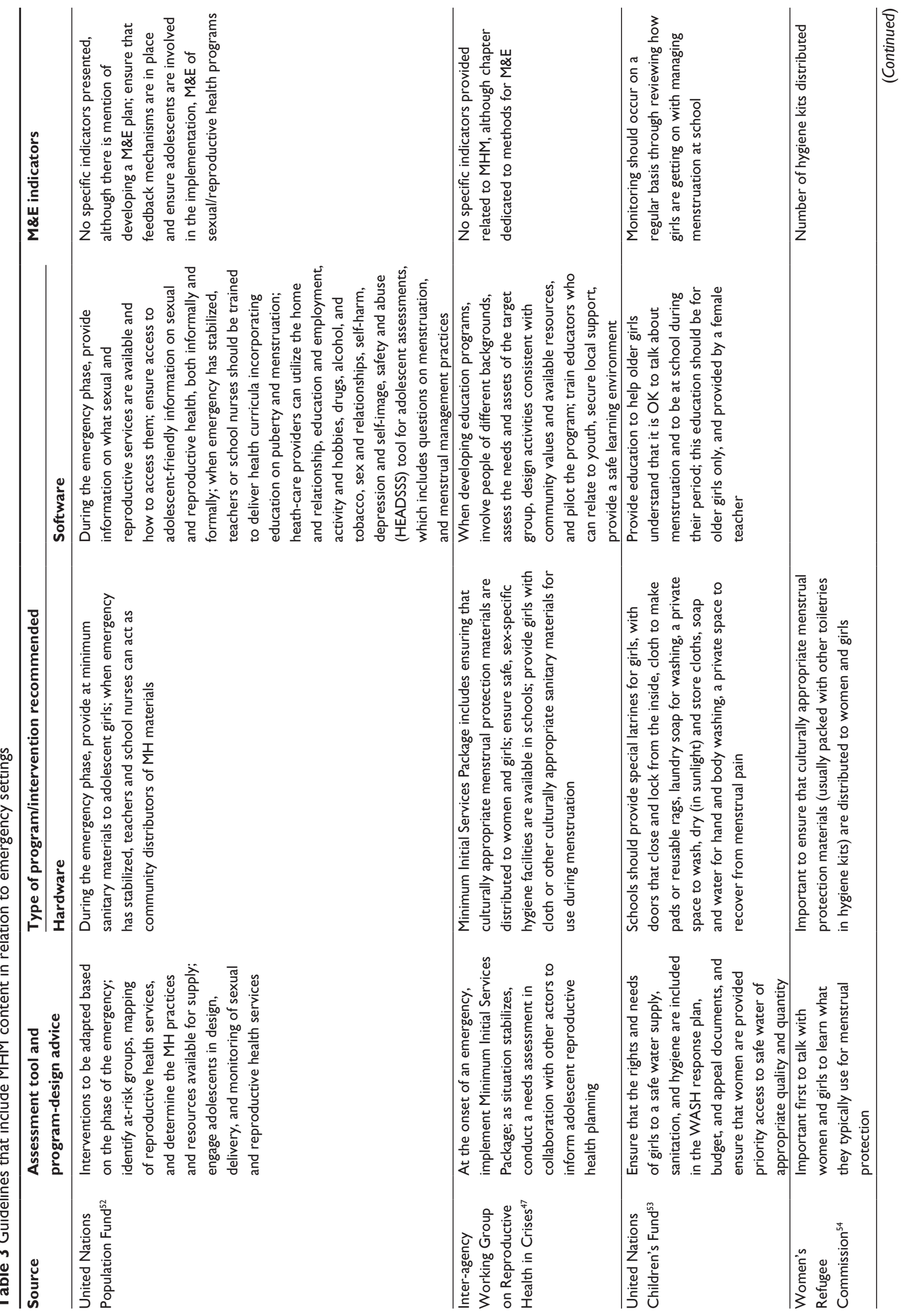




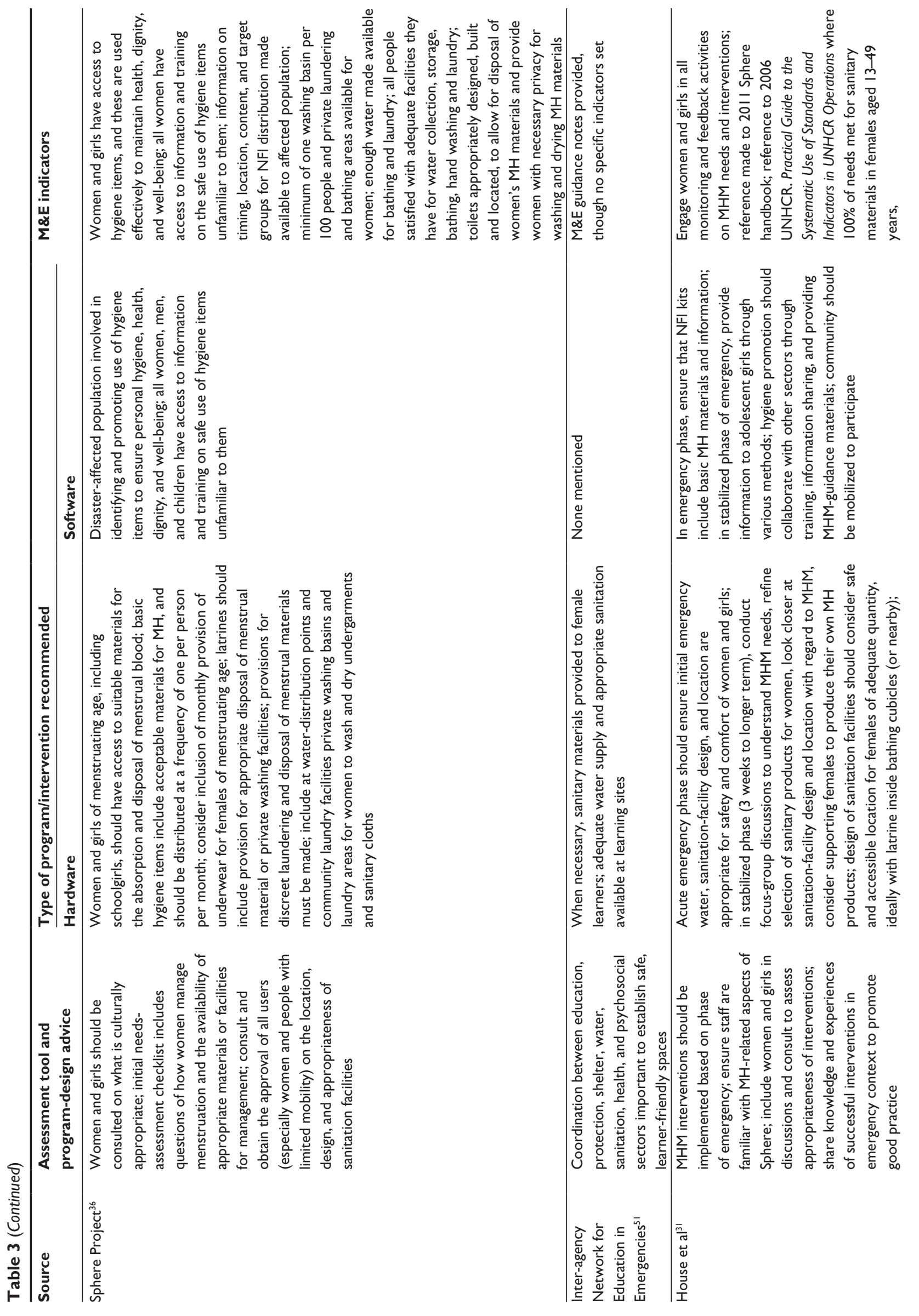



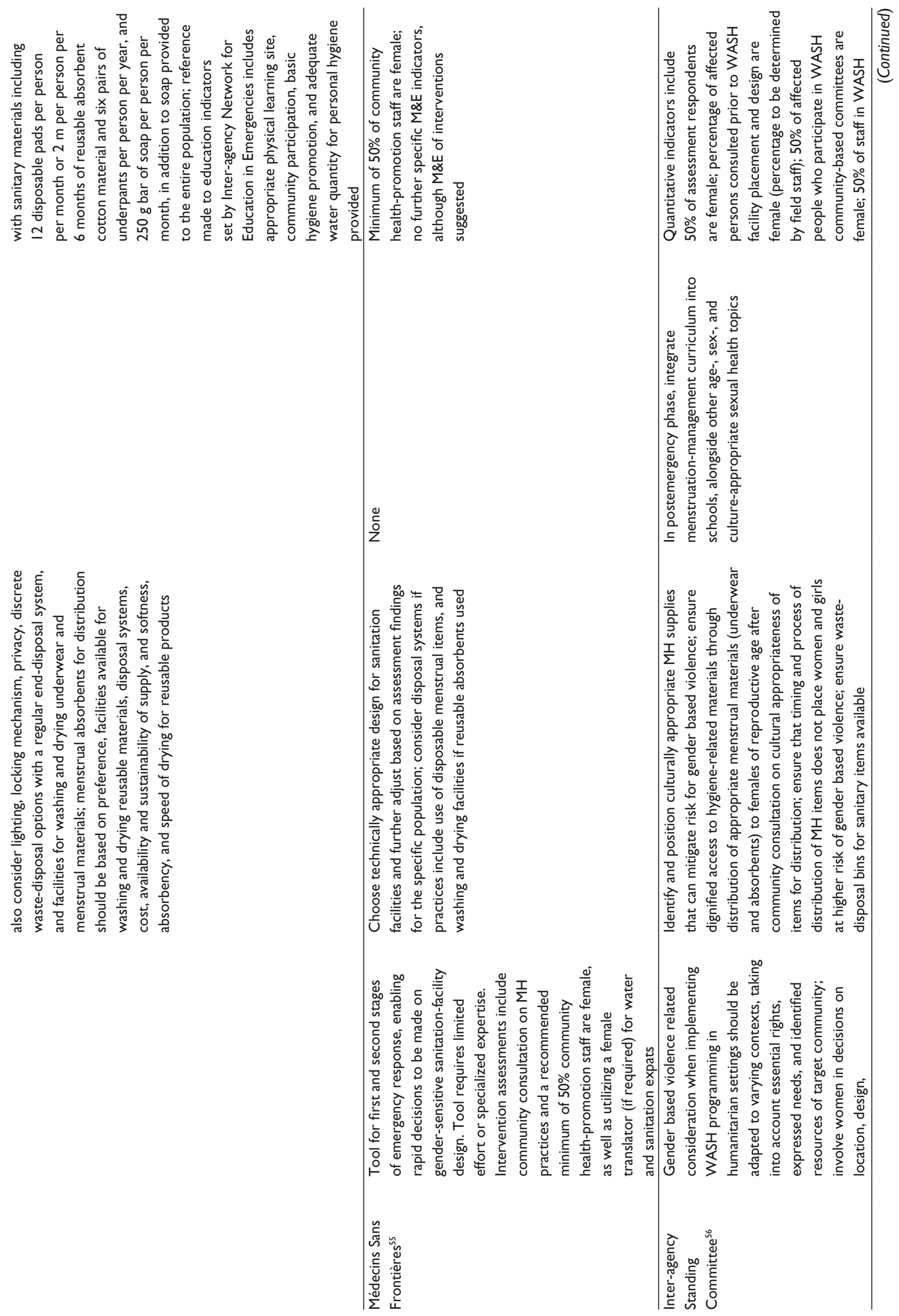


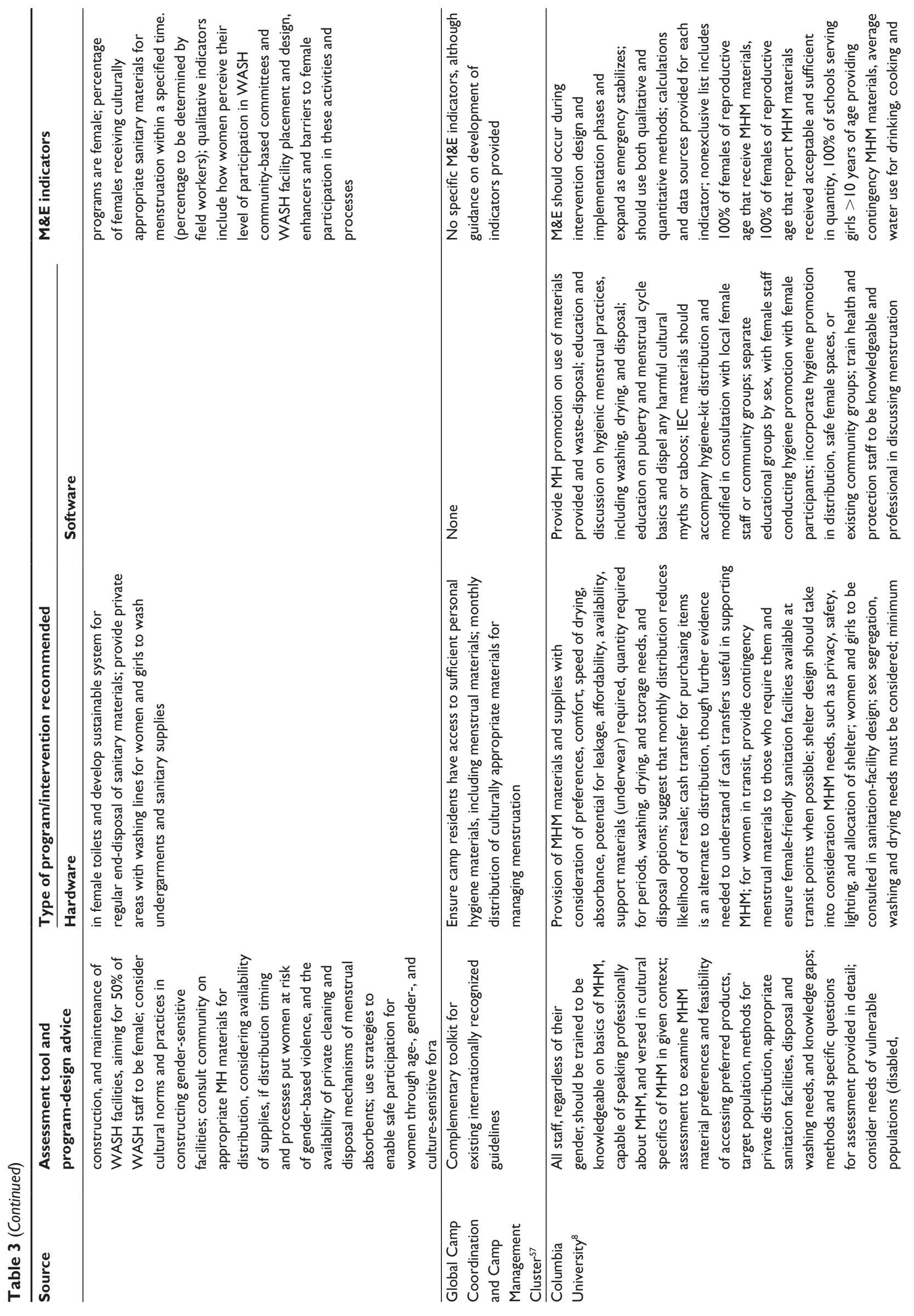



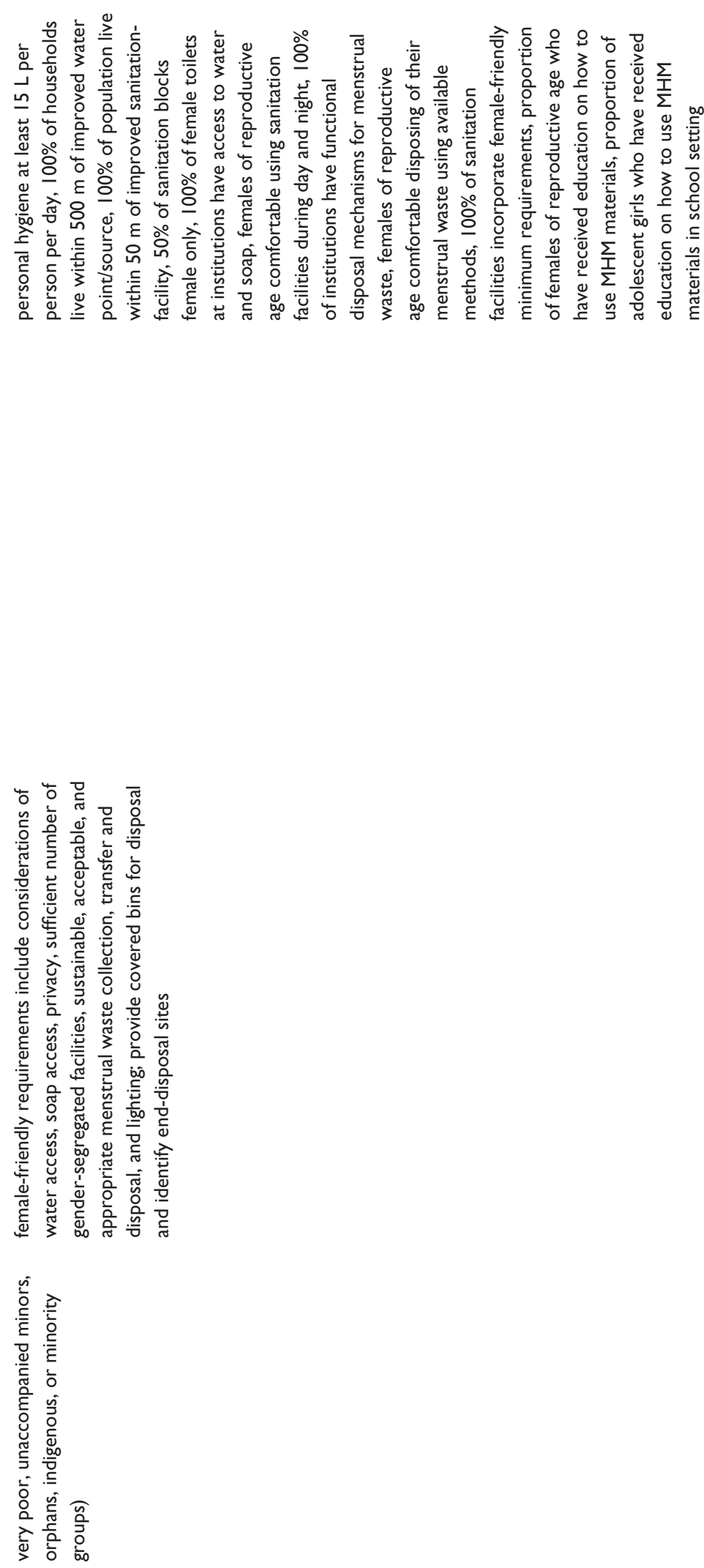
to populations in low-income settings. ${ }^{27} \mathrm{~A}$ lack of empirical evidence examining the introduction and testing of new $\mathrm{MH}$ products, in particular menstrual cups and reusable menstrual underwear, in humanitarian settings was noted.

\section{Sanitation infrastructure}

Along with supportive menstrual materials, an understanding of and action toward the provision of adequate water supply and sanitation facilities that allow for changing in privacy and discrete laundering or disposal of menstrual materials was frequently discussed. $1,3,4,7,12,17,19,24,27,28,31,35,36$ One study ${ }^{25}$ showed how using a quick and easy gender-specific sanitation tool could help design sanitation facilities that were "women-friendly," which includes more privacy for MHM, more space for child care, and increased security. The use of such a tool was found to result in increased use of the facilities after building them in intervention villages.

Considering whether absorbent menstrual products are supported by adequate sanitation facilities was deemed important when designing interventions. ${ }^{4,6,15,19}$ Consulting populations on the appropriateness of both materials and facility design was presented in several policy papers and activity reports..$^{28,31,35-37}$ Several case studies described how consultation with beneficiaries led to improved user satisfaction, although links to health or social outcomes were not made in any of these studies. ${ }^{25,38,39}$ Broad statements about acceptability of products, in particular reusable cloth for displaced populations, ${ }^{21}$ should be reviewed critically, as generalized statements can lead to assumptions that populations do not need to be consulted when designing interventions.

\section{Knowledge and education}

Access to information about menstruation and MH is frequently cited as being important $1^{1,411,27,28}$ and often referred to as "software interventions." ${ }^{40}$ Benner et al ${ }^{17}$ reported that Burmese refugee girls living in camps in Thailand tended to be informed by their mothers about body changes, but apparently little information was given on menstrual issues. Some evidence exists supporting notions that improved menstrual knowledge positively affects MHM and reduces negative psychosocial impact. ${ }^{40}$ Educating populations on how to use products provided in humanitarian responses is important when implementing interventions..$^{19,22,31}$ Incorporating menstrual education into school curricula was a consensus for most addressing this subject, ${ }^{12,19,27}$ and both requires and contributes to overcoming taboos associated with the topic of menstruation.

\section{Health}

Although health outcomes were not the focus of this study and thus not included in the search terms, entries that met all criteria for review did also discuss this topic. One cross-sectional study ${ }^{18}$ among Afghan women presenting with reproductive complaints at basic health units in refugee camps located in Haripur, Pakistan showed that suspected pelvic inflammatory disease was higher in women who used nothing or used an unwashed cloth for managing menstruation compared to women who used a washed cloth to absorb menses ( $p=0.003$ ). However, no adjustment was made for confounding in this paper. Shame, lack of availability of products, inaccessibility due to cost, and prioritizing others' needs over personal menstrual health have all been attributed to unhygienic menstrual practices. ${ }^{7,27,40}$ This was exemplified in one study ${ }^{17}$ that highlighted refugee accounts of wearing underwear still wet from washing, due to the inadequate quantity available to them.

\section{Social context}

Discussion on social impacts of menstruation exists, though there remains a lack of research to quantify outcomes. ${ }^{40}$ Two separate analysis ${ }^{23,41}$ of one qualitative study exploring experiences of sexual embodiment and menstruation in migrants and refugees found recurring themes of shame, secrecy, and dirtiness among the multicultural participants. Additionally, themes of anxiety, fear, and distraction were found to be associated with menstruation. ${ }^{40}$

The link between sanitation infrastructure, menstrual materials, and social impact arose as one author explained how inadequate access to absorbent materials and disposal methods can perpetuate the already existing stigma and shame surrounding menstruation. ${ }^{27}$ Not only have psychosocial stress and a lack of dignity been associated with an inability to manage menstruation effectively, ${ }^{35}$ but depending on the humanitarian setting, needs for managing menstruation can increase risks for female exploitation and sexual violence. ${ }^{6,10}$

Human-rights themes emerged regularly, from declaring MHM an essential aspect of basic human rights ${ }^{12}$ to describing the impact of menstruation on increased sex inequality. ${ }^{1,42}$ According to one study, ${ }^{43}$ taboos with regard to menstruation can have "detrimental effects on the fulfillment of women and girls' human rights, particularly in lowincome settings." The potential consequences for refugee women who have inadequate access to menstrual materials are said to "undermine(s) their opportunity to participate in community activities, registration, food distribution, training programmes, and employment opportunities." 
Conflicting beliefs about menstruation and $\mathrm{MH}$ can exist not only between various cultural groups but also within a single population. This is thought to be attributed to modernizing influences and migration. ${ }^{11}$ Varied findings across studies demonstrate the extensive range of cultural beliefs and confirm the need for understanding and adapting interventions to specific contexts. ${ }^{11}$

Overcoming taboos must start with improved social attitudes and behaviors toward MHM., ${ }^{7,31,44}$ Justifications for including males in these discussions are strong. ${ }^{12,27,45,46}$ It is argued that the taboo that exists toward menstruation has resulted in inadequate acknowledgment of MHM needs in sanitation-system planning and design ${ }^{11}$ and that the responsibility for advocacy and change must be incorporated at all levels: from international organizations and governments down to the private sector and individuals. , 27,31 $^{2}$

\section{Gaps between policy and practice}

Significant progress has been made in advancing refugee rights in the past 20 years, but the need to narrow the gap between MHM policy and practice remains. ${ }^{46}$ A 2013 evaluation ${ }^{20}$ of Minimal Initial Service Package (MISP) activities in a Jordanian refugee camp found inadequate availability of services and supplies for MHM, despite the 2010 addition of an MISP standard stating the need to ensure culturally appropriate MH materials were distributed. ${ }^{47}$ A 2016 review $^{26}$ of MISP activities in postearthquake Nepal echoed these findings. Thapa and Acharya ${ }^{22}$ mentioned that $\mathrm{MH}$ had received low priority, due to the overwhelming need for water, food, and shelter in such crisis situations.

Examining funding can act a proxy indicator of progress. A study ${ }^{16}$ examining reproductive health funding showed increases in the previous 12 years, although that which has been dedicated to MHM was not possible to distinguish, due to the overlap among health, WASH, and nonfood item (NFI) sectors. This corroborates challenges expressed regarding coordination of MHM responses as a multisector issue. ${ }^{6,48}$ The majority of the literature suggested that WASH take the lead on MHM in close collaboration with other relevant sectors. ${ }^{6}$

A lack of systematic documentation can be one contributing factor to the gap between policy and practice, ${ }^{4,6,24}$ which is likely linked to a lack of M\&E of interventions. Organizational experience in MHM interventions exists as seen through the sharing of resources on the public humanitarian platform ReliefWeb. A search for the term "menstrual hygiene" on October 23, 2017 produced 144 Englishlanguage reports written between 1999 and 2017, with increasing frequency in more recent years. Of the 144 returns, 85 reports drew attention to the $\mathrm{MH}-$ related situation in emergencies. Among these, 42 were activity reports written by 22 different organizations who mentioned distribution of $\mathrm{MH}$ products in an emergency context in 25 countries. Thirteen reports discussed sanitation-infrastructure interventions, while only one mentioned the customary practices of the beneficiary community. Though not an empirical representation of progress, the increase in documentation presented does show that MHM is becoming a more frequently reportable topic. As stated in Sommer's 2012 literature review, ${ }^{4}$ it is unfortunate that these brief activity reports do not allow for much in way of learning.

Despite the rarity of learning lessons publicly shared in humanitarian aid reports, some documentation did include recommendations of providing sex-sensitive, participatory responses for MHM, including feedback mechanisms in interventions to create a comfortable environment for women to share information. ${ }^{37,49,50}$ Several reference documents are also publicly accessible, with the most recent, and certainly the most comprehensive, guideline focusing on MHM in emergencies being launched in October 2017.

\section{Current guidelines}

A total of eleven guidelines were identified that discussed MHM in emergency humanitarian contexts. Of these eleven, all but one ${ }^{51}$ specifically mentioned $\mathrm{MH}$ interventions. All guidelines identified were examined for assessment and program-design advice, hardware considerations, software considerations, and M\&E indicators. See Table 3 for an overview of the related information provided in each guideline.

For recommendations related to needs assessment and program design, most documents mentioned the importance of including the targeted beneficiary groups in design, delivery, and monitoring of MHM services, in order to ensure culturally appropriate considerations are made. ${ }^{31,36,52,54-56}$ Consideration of the MHM needs of vulnerable groups (disabled, very poor, unaccompanied minors, orphans, and minority groups) among the wider female population in crisis was recommended in the most recently published guideline. ${ }^{8}$ Several documents mentioned adapting interventions to the context, including the phase of the emergency. ${ }^{31,47,52,55,56}$ Considering timing and location of distribution to promote inclusion and mitigate risk for sex-based violence was also discussed. ${ }^{8,56}$ Collaboration and knowledge sharing with relevant actors in a humanitarian response was found to be important, ${ }^{31,47}$ as was ensuring that staff were aware of and 
competent in discussing MHM needs..$^{8,31}$ There was one mention of ensuring MHM needs were included in budgetary planning. ${ }^{53}$

Hardware interventions focused on the provision of MH materials $8,31,36,47,52,53,57$ and the availability of femalefriendly WASH facilities, including at schools and health facilities. ${ }^{31,36,47,53,55,56}$ Varying degrees of reference were made to specific details of MHM-material access and sanitationfacility design, as noted in Table 3 . House et $\mathrm{al}^{31}$ considered the phases of an emergency in the level of community consultation required before acting, with less in acute phases and increased understanding and revision of programs occurring during stabilization phases. Contrary to this, Médecins Sans Frontières ${ }^{55}$ suggested that community consultation can and should occur in the first and second phases of an emergency, starting with technically appropriate sanitation-facility designs and adjusting these based on assessment findings for the specific beneficiary population.

Software interventions revolved around the access to and provision of education. Depending on the guideline reviewed, more or less emphasis was placed on software interventions. The Inter-agency Network for Education in Emergencies 2012 guideline ${ }^{51}$ provided standards about how to deliver effective education interventions (enhancing the quality of educational preparedness, response and recovery, increasing access to safe and relevant learning opportunities, and ensuring accountability in providing these services); however, there was no mention of any intervention specific to $\mathrm{MH}$. Several recommendations were made to support the success of hardware interventions, such as suggestions that at minimum during the acute emergency phase, basic information on $\mathrm{MH}$, including how to use products distributed and sanitation systems, be provided.,31,36,52 Several guidelines recommended facilitating female-only groups for education sessions. . $^{8,31,53}$

$M \& E$ indicators were lacking in many of the documents, although guidance notes for developing M\&E plans were mentioned. Guidelines that did provide specific indicators focused on access to adequate sanitation materials and facilities, sex inclusion in programming, education access and knowledge acquisition, and quantifiable indicators, such as number of menstrual materials distributed or received by the target population, ${ }^{31,54,56}$ method of item distribution, ${ }^{36}$ quantity and appropriateness of water and sanitation facilities available, ${ }^{8}$ access to and participation in educational activities or materials, ${ }^{8,53}$ and the engagement of female staff or community participants in various phases of programming, ${ }^{31,55,56}$ were included alongside qualitative indicators. Qualitative indicators examined user satisfaction with sanitation facilities, ${ }^{8,36}$ perceptions of levels of participation in programming, and barriers to participation in such groups. ${ }^{56}$

Until recently, House et $\mathrm{al}^{31}$ had presented the most comprehensive document on MHM in emergencies, with "knowledge and practice on $\mathrm{MH}$ programming from around the world to encourage the development of comprehensive and context specific approaches to MH.” In October 2017, a significant addition to MHM guidelines in emergency settings was launched by Columbia University and the International Rescue Committee. ${ }^{8}$ As a document dedicated to filling the identified gaps, the authors collated all information on assessment, best practices, and M\&E indicators. The information was supported by case studies and developed as a cross-sector tool for use by the various sectors involved in MHM responses.

\section{Discussion}

This review included 51 articles that discussed MHM in emergency contexts (current policies, guidelines, empirical research, and humanitarian aid activities addressing populations' menstrual needs): 16 articles were peer-reviewed papers and 35 gray literature. Nine of the peer-reviewed papers were original research studies and seven literature reviews. From the gray literature, four were master's theses, 11 guidelines, and $20 \mathrm{NGO}$ reports (communications, policy briefs, evaluations, and case studies).

From the reviews found in the search, only two focused in MHM in emergency context. This review aimed to extend and update the existing information included in these two pieces of work. However our intention was not to provide a critical appraisal of the included studies, but a summary of issues for consideration and consensus in approaches to addressing MHM in emergency contexts.

There is consistency throughout the literature about the components required in MHM; however, there is a lack of evidence on the application of interventions for these in the field. Dignity kits often include both reusable items, such as clothing, buckets, and shoes, as well as disposal materials, such as sanitary pads. Lacking in many guidelines are statements of renewing supplies on a monthly basis. This is supported by narratives from displaced populations that state that they received sufficient items on arrival, but maintenance was inadequate to meet needs. ${ }^{49}$ There is also consistent agreement about the type of materials that should be distributed and how important it is to assess women and girls needs before designing an intervention in each 
different setting. However, in most of the case studies in this review, the organizations tended to distribute mainly disposable menstrual materials.

The potential introduction of new menstrual products in emergency settings was suggested in some of the literature; however, no studies about new products were found with a focus on humanitarian contexts. Two studies examining the acceptability and utility of menstrual cups in low-income settings were identified. In Kenya, ${ }^{58}$ a mixed-method study collected baseline data and compared this to data 4 months after introducing menstrual cups. In Zimbabwe, ${ }^{59}$ hypothetical acceptability was examined through a qualitative study. In both studies, links were made among product utility, product cost, sanitation infrastructure, and education provided on proper use. Results in both studies showed a willingness either to try or continue to use the product. The lack of studies conducted in humanitarian contexts can be justified when considering the urgency of life-saving needs.

The importance of "women-friendly" sanitation facilities that can offer a comfortable space for the female population to deal with their menstruation was also highly recognized. Having a proper needs assessment including the female community before designing appropriate sanitation facilities is an essential duty of humanitarian agencies. ${ }^{60,61}$ It is also recognized that sometimes in an emergency, there is no time for a proper participatory assessment, but some examples in the literature ${ }^{25}$ have shown that even quick and easy tools could be feasible to implement (without incurring implementation delays), and they can have great impact.

Educating populations about the importance of MHM and girls and women about how to use menstrual products provided in humanitarian responses is important when implementing interventions. More education about physiological changes that adolescents face when reaching puberty is also important to help them to welcome this special moment. There was lots of agreement about incorporating part of this training and education within the school system, ${ }^{61}$ but some papers also suggested the importance to work within families ${ }^{17}$ or the creation of counseling services, psychological support groups, and women's self-help groups, which could have a big impact on the well-being of girls and women in times of natural disasters. ${ }^{22}$ Two systematic reviews assessing the association of the social effects of MHM concluded that there was good evidence that educational interventions can improve MHM practices and reduce social restrictions, but they also concluded that there is a lack of strong evidence and that the studies included presented a high risk of bias, mainly due to outcome assessment. 9,40
Only one peer-reviewed paper ${ }^{18}$ was found in this search that assessed the association between MHM practices and reproductive infections. This article showed that unhygienic practices were associated with pelvic infection. In additional literature, there was no strong evidence to show which type of material and menstrual practices were associated with health impact. One systematic review ${ }^{9}$ examined both health and social effects of MHM after this gap in research had been identified in 2012. ${ }^{7}$ Minimal evidence available showed inconclusive links between MHM and negative health outcomes, ${ }^{9}$ although it has been stated that "It is biologically plausible that unhygienic MHM practices can affect the reproductive tract but the specific infection, the strength or the effect, and the route of transmission, remain unclear." 12

Experiences and constructions of menstruation are shaped by the sociocultural environment in which women are embedded. Cultural, societal, religious, and other factors usually influence a women's experience in this sphere. Humanitarian contexts can result or increase the negative experiences that women suffer related to menstruation (among other things). Perceptions of anxiety, fear, shame, and distraction are found to be associated with menstruation. An article published by Thomson Reuters Foundation ${ }^{62}$ describes the challenges that refugee women face in managing menstruation. The narratives of Syrian refugees describe how the lack of sanitary products created a sense of isolation and fear. Many other factors associated with these experiences have also been reported in these contexts (barriers to access of safe and private sanitation facilities, lack of support by international agencies, stress when having to adapt to new menstrual management practices).

In the last few years, global attention to integrate MHM into humanitarian response guidelines has increased. Special attention to MHM-product distribution and construction and adaptation of "women-friendly" WASH facilities has been given. Specifying the quantity of menstrual products to provide to females of menstruating age in guidelines can be useful as a reference, although considering the diversity of practices among various populations, as well as within populations, ${ }^{11}$ these specifications can also undermine the importance of consulting beneficiaries to understand their specific needs. Indicators set out in the most recent toolkit for MHM in emergencies ${ }^{8}$ provided space for program adaptation through considering female reports of receiving adequate and sufficient materials to meet their specific needs. Assessing beneficiaries' needs was also an important factor to consider, mentioned in almost all the guidelines. However, it has been 
noted that cultural taboos regarding discussing MHM with beneficiaries may impact the comfort of staff; therefore, more effort should be made to prepare and train competent staff in this area.

Despite the progress seen in the last few years, many gaps still exist, such as agreement about which indicators can be used to monitor and evaluate MHM interventions, lack of clarity about how to identify key components of an MHM response in different emergency settings, which actors should be integrated, and clear guidelines to assess context and beneficiaries' needs. A review ${ }^{13}$ also highlighted the need for systematic approaches or guidelines to issues of inclusiveness in the emergency context. As a response to most of these gaps, Columbia University has just published a toolkit ${ }^{8}$ that aims to provide streamlined guidance to support organizations and agencies seeking to integrate $\mathrm{MHM}$ rapidly into existing programming across sectors and phases.

In conclusion, there is much still to be done to build the evidence base that will feed guidelines and programs to improve MHM among girls and women in emergency contexts. With this work, we hope we have provided some basis for those planning future research in this area. We believe that there is much scope for dedicated $\mathrm{MH}$ research in emergency contexts, and more energy and resources should be invested by NGOs in measuring and reflecting about the failure and success of their programs.

\section{Recommendations}

- Consultation with girls and women before designing and implementing hardware and software interventions

- hardware interventions should include not only type of absorbent but also other menstrual support items (soap, underwear, torches, and wash basins); they should also focus on providing adequate WASH facilities that will cover needs (space, privacy, location, distribution, disposal, and access of water)

- software interventions should include training in the use of menstrual materials, education of both boys and girls in menstrual biology and hygiene, and explore new initiatives to create a better social-support environment (counseling and self-help groups)

- when designing hardware and software interventions, consider issues of sustainability, equity, and inclusion

- invest time and resources in training competent staff responsible for assessment and delivery of MHM interventions

- intervention-delivery channels should be researched
- invest more time and thinking into how to coordinate responses among different actors and sharing of experiences (failures and successful attempts)

- adjust indicators based on expressed needs in relation to cultural and customary practices

- test tools for designing interventions and evaluation of their effectiveness

- monitor and evaluate interventions taking place in the humanitarian context and sharing of results among the humanitarian community

- evidence-based research should feed improvements for MHM guidelines.

\section{Disclosure}

The authors report no conflicts of interest in this work.

\section{References}

1. WHO/UNICEF Joint Monitoring Program for Water Supply and Sanitation. Consultation on draft long list of goal, target and indicator options for future monitoring of water, sanitation and hygiene. 2012. Available from: http://www.rural-water-supply.net/en/resources/details/378. Accessed January 28, 2018.

2. Sommer M, Chandraratna S, Cavill S, Mahon T, Phillips-Howard P. Managing menstruation in the workplace: an overlooked issue in lowand middle-income countries. Int Jr Equity Health. 2016;15:86-90.

3. Sommer M, Hirsch J, Nathanson C, Parker R. Comfortably, safely, and without shame: defining menstrual hygiene management as a public health issue. Am J Public Health. 2015;105:1302-1311.

4. Sommer M. Menstrual hygiene management in humanitarian emergencies: gaps and recommendations. Waterlines. 2012;31:83-104.

5. United Nations High Commissioner for Refugees. Global Trends: Forced Displacement in 2016. Geneva: UNHCR; 2017.

6. Sommer M, Schmitt ML, Clatworthy D, Bramucci G, Wheeler E, Ratnayake R. What is the scope for addressing menstrual hygiene management in complex humanitarian emergencies? Waterlines. 2016;35: 245-264.

7. Biran A, Curtis V, Gautam OP, et al. Background paper on measuring WASH and food hygiene practices: definition of goals to be tackling post-2015 by the Joint Monitoring Programme. Available from: https:// www.ircwash.org/resources/background-paper-measuring-washand-food-hygiene-practices-definition-goals-be-tackled. Accessed January 28, 2018.

8. Columbia University. A toolkit for integrating menstrual hygiene management (MHM) into humanitarian response: the full guide. 2017. Available from: https://reliefweb.int/sites/reliefweb.int/files/resources/ mhm-emergencies-toolkit-full_0.pdf. Accessed January 28, 2018.

9. Sumpter C, Torondel B. A systematic review of the health and social effects of menstrual hygiene management. PLoS One. 2013;8:e62004.

10. Sommer M, Ferron S, Cavill S, House S. Violence, gender and WASH: spurring action on a complex, under-documented and sensitive topic. Environ Urban. 2015;27:105-116.

11. Sommer M, Kjellen M, Pensulo C. Girls' and women's unmet needs for menstrual hygiene management (MHM): the interactions between MHM and sanitation systems in low-income countries. J Water Sanit Hyg Dev. 2013;3:283-297.

12. Balls E. Menstrual hygiene management. 2017. Available from: http:// menstrualhygieneday.org/wp-content/uploads/2017/01/SHARE_MHM_policybrief_2017.pdf. Accessed January 28, 2018. 
13. Brown J, Cavill S, Cumming O, Jeandron A. Water, sanitation, and hygiene in emergencies: summary review and recommendations for future research. Waterlines. 2012;31:11-29.

14. Gul M, Gul E, Gilbert B. Studying detriments to WASH response in vulnerable women populations during flood emergencies in Pakistan: a review of interventions through a climate risk lens. Proceedings of the National Symposium on Climate Change Vulnerability Mapping of Water Resources of Pakistan; May 30th, 2016; Islamabad, Pakistan.

15. Ersel M. Water and sanitation standards in humanitarian action. Turk J Emerg Med. 2015;15:27-33.

16. Tanabe M, Schaus K, Rastogi S, Krause S, Patel P. Tracking humanitarian funding for reproductive health: a systematic analysis of health and protection proposals from 2002-2013. Confl Health. 2015; 9:S2.

17. Benner M, Townsend J, Kaloi W, et al. Reproductive health and quality of life of young Burmese refugees in Thailand. Confl Health. 2010;4:5.

18. Balsara ZP, Wu I, Marsh DR, et al. Reproductive tract disorders among Afghan refugee women attending health clinics in Haripur, Pakistan. J Health Popul Nutr. 2010;28:501-508.

19. Parker AH, Smith JA, Verdemato T, Cooke J, Webster J, Carter RC Menstrual management: a neglected aspect of hygiene interventions. Disaster Prev Manag. 2014;23:437-454.

20. Krause S, Williams H, Onyango MA, et al. Reproductive health services for Syrian refugees in Zaatri Camp and Irbid City, Hashemite kingdom of Jordan: an evaluation of the Minimum Initial Services Package. Conf Health. 2015;9:S4

21. Budhathoki SS, Bhattachan M, Pokharel P, Bhadra M, van Teijlingen E. Reusable sanitary towels: promoting menstrual hygiene in post-earthquake Nepal. J Fam Plann Reprod Health Care. 2017;43: $157-159$.

22. Thapa SB, Acharya G. Women's health is not in focus in disaster zones: lessons from the Nepal earthquake. J Fam Plann Reprod Health Care. 2017;43:92-93.

23. Hawkey AJ, Ussher JM, Perz J, Metusela C. Experiences and constructions of menarche and menstruation among migrant and refugee women. Qual Health Res. 2017;27:1473-1490.

24. Schmitt ML, Clatworthy D, Ratnayake R, et al. Understanding the menstrual hygiene management challenges facing displaced girls and women: findings from qualitative assessments in Myanmar and Lebanon. Confl Health. 2017;11:19.

25. de Lange R, Lenglet A, Fesseletet JF, et al. Keeping it simple: a genderspecific sanitation tool for emergencies. Waterlines. 2014;33:45-54.

26. Krause S. Evaluation of the MISP for Reproductive Health Services in Post-Earthquake Nepal. New York: Women's Refugee Commission; 2016.

27. Swedish International Development Cooperation Agency. Health: both a prerequisite and an outcome of sustainable development. 2016 Available from: https:/www.sida.se/contentassets/2d05faf3aebc4092 a0ef96439c026262/18565.pdf. Accessed January 29, 2018.

28. Rohwerder B. Non-food items (NFIs) and the needs of women and girls in emergencies. 2014. Available from: http://www.ids.ac.uk/ publication/non-food-items-nfis-and-the-needs-of-women-and-girlsin-emergencies. Accessed January 29, 2018.

29. United Nations Population Fund. Female dignity kits: making a difference for women and girls in Myanmar. Available from: https://myanmar. unfpa.org/sites/default/files/pub-pdf/Dignity_kits_EN_v1_07August_0. pdf. Accessed January 29, 2018.

30. Ferron S. Enabling Access to Non-Food Items in an Emergency Response: A Review of Oxfam Programmes. Oxford: Oxfam; 2017.

31. House S, Mahon T, Cavill S. Menstrual hygiene matters. 2012. Available from: https://washmatters.wateraid.org/publications/menstrualhygiene-matters. Accessed January 29, 2018.

32. Keith B. Girls' and women's right to menstrual health: evidence and opportunities. 2016. Available from: https:/www.path.org/publications/ files/RH_outlook_mh_022016.pdf. Accessed January 29, 2018.
33. Musaazi MK, Mechtenberg AR, Nakibuule J, et al. Quantification of social equity in life cycle assessment for increased sustainable production of sanitary products in Uganda. J Clean Prod. 2015;96:569-579.

34. Israr H, Nasir SS. Low cost handmade sanitary pads! From design to production: a step forward in menstrual hygiene promotion in Pakistan. 2012. Available from: https://sanitationupdates.files.wordpress.com/2012/07/menstrual_hygiene_pakistan20121.pdf. Accessed January 29, 2018.

35. Mills JE, Cumming O. The impact of water, sanitation and hygiene on key health and social outcomes: review of evidence. 2016. Available from: https://www.unicef.org/wash/files/The_Impact_of_WASH_ on_Key_Social_and_Health_Outcomes_Review_of_Evidence.pdf. Accessed January 29, 2018.

36. Sphere Project. Humanitarian Charter and Minimum Standards in Disaster Response. 3rd ed. Rugby, UK: Practical Action Publishing; 2011.

37. Cooperative for Assistance and Relief Everywhere. Rapid gender and protection assessment report: Kobane refugee population, Suruç, Turkey. 2014. Available from: https://reliefweb.int/report/turkey/rapidgender-and-protection-assessment-report-kobane-refugee-populationsuruc-turkey. Accessed January 29, 2018.

38. Wicken J, Verhagen J, Sijbesma C, Da Silva C, Ryan P. Beyond Construction: Use by All. London: WaterAid; 2008.

39. Rangaswami S. Ideas That Work: A Gender, WASH, and Emergencies Toolkit. Oxford: Oxfam; 2010.

40. Hennegan J, Montgomery P. Do menstrual hygiene management interventions improve education and psychosocial outcomes for women and girls in low and middle income countries? A systematic review. PLoS One. 2016;11:e0146985.

41. Ussher JM, Perz J, Metusela C, et al. Negotiating discourses of shame, secrecy, and silence: migrant and refugee women's experiences of sexual embodiment. Arch Sex Behav. 2017;46:1901-1921.

42. Water Supply and Sanitation Collaborative Council. Annual Report: 2016. Geneva: WSSCC; 2017.

43. Boosey R, Wilson E. A vicious cycle of silence: what are the implications of the menstruation taboo for the fulfillment of women and girls' human rights, and to what extent is the menstruation taboo addressed by international human rights law and human rights bodies? 2014. Available from: http://eprints.whiterose.ac.uk/80597/1/A\%20vicious\%20 cycle \%20of\%20silence\%20white\%20rose\%20report.pdf. Accessed January 29, 2018.

44. United Nations Secretary-General's Advisory Board on Water and Sanitation. The UNSGAB Journey. New York: UNSGAB; 2015.

45. Lanz K, Bieri S, Fankhauser L. Critical gender issues with regard to food, land, and water: a compendium for policy-makers, NGOs, and researchers. 2012. Available from: http://www.izfg.unibe.ch/unibe/ portal/center_generell/title_fak_ueberg/izfg/content/e7734/e7735/ e7736/e87950/files87975/CriticalGenderIssueswithRegardtoFoodLandandWater_ger.pdf. Accessed January 29, 2018.

46. Buscher D. Refugee women: twenty years on. Refug Surv Q. 2010; 29:4-20.

47. Inter-agency Working Group on Reproductive Health in Crises. Interagency Field Manual on Reproductive Health in Humanitarian Settings: 2010 Revision for Field Review. Geneva: IAWG; 2010.

48. Travers CL. No One Cares We're Bleeding: The Place of Menstrual Management In Humanitarian Response [master's thesis]. Uppsala, Sweden: Uppsala University; 2015.

49. Djibouti Red Crescent Society. Djibouti/Yemen unrest. 2015. Available from: https://reliefweb.int/sites/reliefweb.int/files/resources/ Djibouti\%20DREF\%20Review\%20Final\%20Report.pdf. Accessed January 29, 2018.

50. West Bengal State Inter Agency Group. Report of joint rapid need assessment: south Bengal flood 2017. 2017. Available from: https:// reliefweb.int/sites/reliefweb.int/files/resources/jrna-report-of-southbengal-flood-2017.pdf. Accessed January 29, 2018. 
51. Inter-agency Network for Education in Emergencies. Minimum Standards for Education: Preparedness, Response, Recovery. 2nd ed. New York: INEE; 2012.

52. United Nations Population Fund. Adolescent Sexual and Reproductive Health Toolkit of Humanitarian Settings: A Companion to the Interagency Field Manual on Reproductive Health in Humanitarian Settings. New York: UNFPA; 2009.

53. United Nations Children's Fund. Water, Sanitation, and Hygiene for Schoolchildren in Emergencies: A Guidebookfor Teachers. New York: UNICEF; 2011.

54. Women's Refugee Commission. Minimum initial service package (MISP) for reproductive health in crisis situations: a distance learning module. 2011. Available from: https://reliefweb.int/report/world/ minimum-initial-service-package-misp-reproductive-health-crisissituations-distance. Accessed January 29, 2018.

55. Médecins Sans Frontières. Gender and sanitation tool for displaced populations. 2014. Available from: http://fieldresearch.msf.org/msf/ bitstream/10144/336731/1/Gender+and+Sanitation+Tool+Final+ Dec+8+2013+(1).pdf. Accessed January 29, 2018.

56. Inter-agency Standing Committee. Guidelines for Integrating GenderBased Violence Interventions in Humanitarian Action: Reducing Risk, Promoting Resilience, and Aiding Recovery. New York: IASC; 2015.
57. Global Camp Coordination and Camp Management Cluster. Camp Management Toolkit. Geneva: International Organization for Migration; 2015.

58. African Population and Health Research Center. Attitudes Towards, and Acceptability of, Menstrual Cups as a Method for Managing Menstruation: Experiences of Women and Schoolgirls in Nairobi, Kenya. Nairobi: APHRC; 2010.

59. Averbach S, Sahin-Hodoglugil N, Musara P, Chipato T, van der Straten A. Duet for menstrual protection: a feasibility study in Zimbabwe. Contraception. 2009;79:463-468.

60. Rohwerder B. Women and girls in forced and protracted displacement. 2016. Available from: http://www.gsdrc.org/wp-content/uploads/ 2016/06/HDQ1364.pdf. Accessed January 29, 2018.

61. Inter Agency Group Assam. Joint needs assessment report: Assam floods 2017. 2017. Available from: https://reliefweb.int/sites/reliefweb. int/files/resources/joint-needs-assessment-report_assam-floods_2017_ final.pdf. Accessed January 29, 2018.

62. Pujol-Mazzini A. For refugee women, periods a dangerous, shameful time. 2017. Available from: http://news.trust.org/item/20170308150652db2y1. Accessed January 29, 2018.
International Journal of Women's Health

\section{Publish your work in this journal}

The International Journal of Women's Health is an international, peerreviewed open-access journal publishing original research, reports, editorials, reviews and commentaries on all aspects of women's healthcare including gynecology, obstetrics, and breast cancer. The manuscript management system is completely online and includes

\section{Dovepress}

a very quick and fair peer-review system, which is all easy to use. Visit http://www.dovepress.com/testimonials.php to read real quotes from published authors. 